\title{
Semiconducting defect-free polymorph of borophene: Peierls distortion in two dimensions
}

\author{
S. İpek, ${ }^{1}$ M. E. Kilic, ${ }^{2}$ A. Mogulkoc, ${ }^{3}$ S. Cahangirov, ${ }^{2}$ and E. Durgun ${ }^{2, *}$ \\ ${ }^{1}$ Department of Engineering Physics, Istanbul Medeniyet University, Istanbul 34700, Turkey \\ ${ }^{2}$ UNAM - National Nanotechnology Research Center and Institute of Materials Science and Nanotechnology, Bilkent University, \\ Ankara 06800, Turkey \\ ${ }^{3}$ Department of Physics, Faculty of Sciences, Ankara University, 06100 Tandogan, Ankara, Turkey
}

(Received 17 August 2018; published 17 December 2018)

\begin{abstract}
In contrast to the well-defined lattices of various two-dimensional (2D) systems, the atomic structure of borophene is sensitive to growth conditions and type of the substrate which results in rich polymorphism. By employing $a b$ initio methods, we reveal a thermodynamically stable borophene polymorph without vacancies which is a semiconductor unlike the other known boron sheets, in the form of an asymmetric centered-washboard structure. Our results indicate that asymmetric distortion is induced due to Peierls instability which transforms a symmetric metallic system into a semiconductor. We also show that applying uniaxial or biaxial strain gradually lowers the obtained band gap and the symmetric configuration is restored following the closure of the band gap. Furthermore, while the Poisson's ratio is calculated to be high and positive in the semiconducting regime, it switches to negative once the metallicity is retrieved. The realization of semiconducting borophene polymorphs without defects and tunability of its electronic and mechanical response can extend the usage of boron sheets in a variety of nanoelectronic applications.
\end{abstract}

DOI: 10.1103/PhysRevB.98.241408

Boron has a short covalent radius, owns delocalized electrons, and can adopt $s p^{2}$ hybridization as does carbon, which leads to the formation of numerous low-dimensional structures, including nanotubes [1], fullerenes [2,3], and clusters [4]. Following the realization of quasiplanar small boron clusters [5,6], various two-dimensional (2D) boron sheets, namely borophene [5,7], with similar cohesive energies are also predicted. The vacancies [8] and/or out-of-plane distortions [9] have been shown to enhance the stability of these structures. In parallel with theoretical predictions, closed-packed, triangular buckled [10], and planar triangular polymorphs of borophene with different vacancy patterns [11] are grown on $\operatorname{Ag}(111)$ substrates. Very recently, the coexistence of two distinct polymorphs of borophene with line defects have been revealed experimentally on $\operatorname{Ag}(111)$ substrates [12].

Depending on its structure and geometry, borophene possesses novel properties which differ significantly from its bulk counterparts. For instance, while bulk structures of boron are semiconductors, borophene polymorphs [13] are metallic [10-12]. Recently, two phases of borophene $\left(\beta_{12}\right.$ and $\chi_{3}$ sheets) have been predicted to have phonon-mediated superconductivity with a considerably high critical temperature [14]. $\beta_{12}$-borophene also hosts gapless Dirac cones mainly contributed by the $p_{z}$ orbital of boron [15]. This prediction is further confirmed experimentally based on angleresolved photoemission spectroscopy (ARPES) results [15]. The buckled phase of borophene ( $\delta_{6}$-borophene) has corrugated rows of atoms in one lattice direction, resulting in strong anisotropy in the electronic $[10,16]$, mechanical $[10,17]$, thermal $[18,19]$, and optical properties $[16,20]$. Owing to the

\footnotetext{
*durgun@unam.bilkent.edu.tr
}

peculiar properties of borophene allotropes, these systems are promising candidates to be used in various technological applications including flexible optically transparent electrode devices [21,22], electrocatalysts for hydrogen evolution reactions [23], thermal management applications for efficient heat transport [24], and electronic junctions based on selfassembled heterostructures [25].

In contrast to the well-defined lattices of various 2D systems, borophene can exhibit a variety of polymorphs resulting from both intrinsic factors such as a multicenter bonding scheme of boron atoms and extrinsic factors including substrate type and growth conditions [13]. Accordingly, different polymorphs of borophene with similar cohesive energies [7] but with distinct properties can be obtained. Motivated by the recent advances in borophene, in this Rapid Communication we explore rare borophene polymorphs without vacancies/defects, and show that a thermodynamically stable, asymmetric centered-washboard structure which is similar to the geometry of monolayer black phosphorus can be obtained. Following the analysis of the structural properties, we reveal the origin of the asymmetric distortion (Peierls instability) which transforms a metallic system into a semiconductor by opening a gap in the electronic band structure. We also examine the electronic and mechanical response of the system to applied uniaxial or biaxial strain and obtain a semiconductor to metal phase transition together with a positive to negative Poisson's ratio alteration.

We carried out first-principles calculations based on spinpolarized density functional theory (DFT) $[26,27]$ implemented in the Vienna $a b$ initio simulation package (VASP) [28]. Projector augmented-wave (PAW) potentials [29] with a kinetic energy cutoff of $420 \mathrm{eV}$ for the plane-wave basis set were used. The generalized gradient approximation (GGA) 
(a)
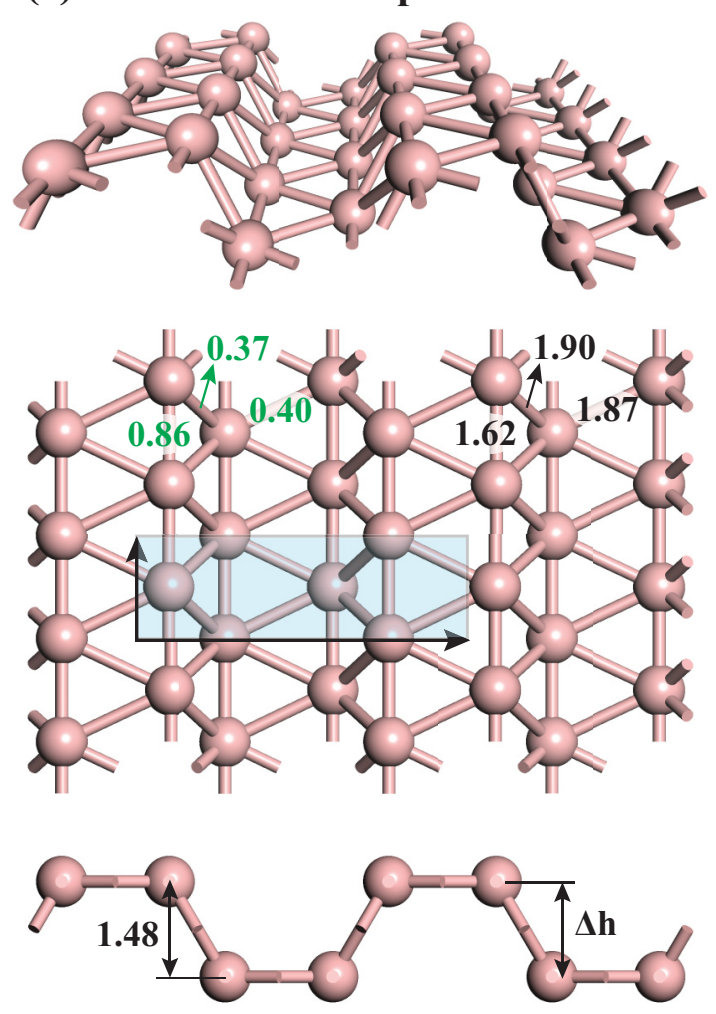
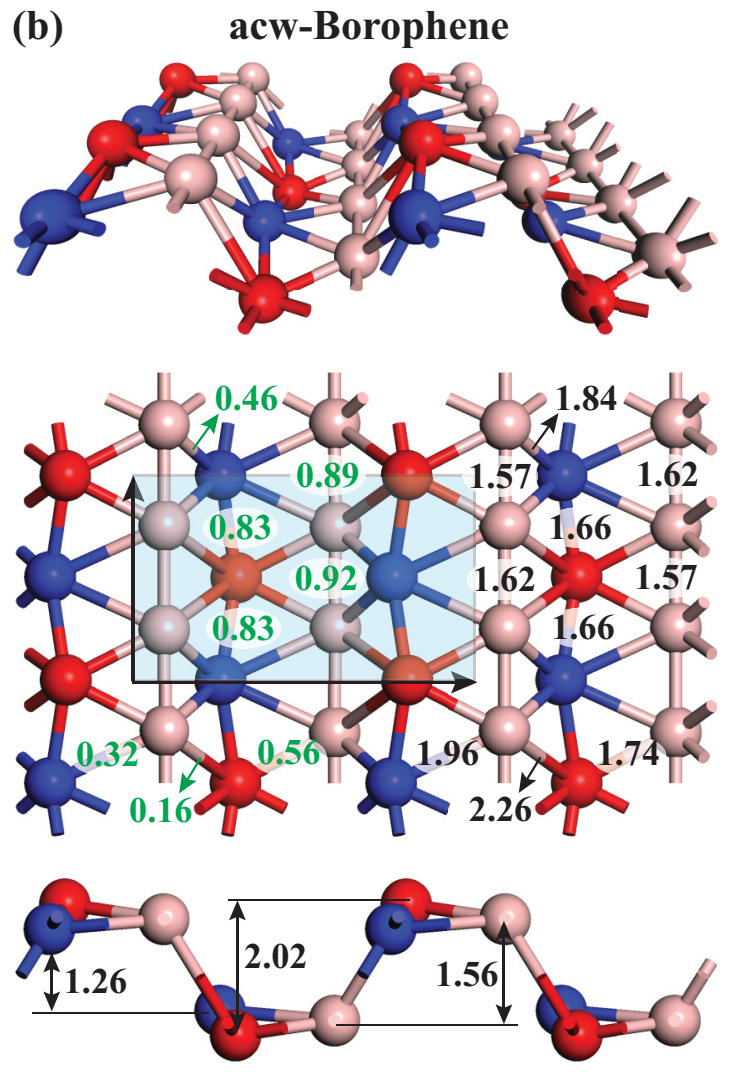

FIG. 1. Perspective, top, and side views of the optimized atomic structures of (a) centered-washboard (cw) and (b) asymmetric centeredwashboard (acw) borophene, respectively. The unit cells are shown by shaded rectangles. Bond orders (green), puckering height $(\Delta h)$, and bond lengths (black) are indicated. Blue and red spheres emphasize the distorted boron atoms.

within the Perdew, Burke, and Ernzerhof (PBE) scheme [30] was utilized to describe the exchange-correlation potential. The electronic band-structure calculations were also calculated with Heyd-Scuseria-Ernzerhof hybrid functionals (HSE06) [31] to correct the underestimated band-gap values. All atomic positions and lattice constants are optimized using the conjugate gradient method without any constraints by setting the convergence criteria on the total energy and force to $10^{-5} \mathrm{eV}$ and $10^{-2} \mathrm{eV} / \AA$, respectively. A $\Gamma$-centered $20 \times$ $16 \times 1 k$-point mesh is used for the Brillouin zone integrations of the primitive unit cell and then scaled accordingly with the cell size. A supercell geometry with a vacuum space of $15 \AA$ in the nonperiodic direction was adopted to avoid spurious interactions between the periodic images. The stability of the obtained structures was tested with a phonon spectrum analysis using density functional perturbation theory (DFPT) [32] and high-temperature $a b$ initio molecular dynamic (MD) calculations considering a microcanonical ensemble [28].

A large variety of borophene polymorphs with vacancies have been reported and it has been shown that the stability of these systems is mainly provided by vacancy patterns $[7,11,33]$. On the other hand, ideal borophene crystals without defects are scarce $[10,34]$. Motivated by the buckled structure of $\delta_{6}$-borophene (belonging to the Pmmn space group) where stability is enhanced by out-of-plane distortions $[9,10]$ instead of vacancies, we look for the possibility of obtaining ideal washboard structures of borophene which are not planar but have puckered geometries. Starting with various initial configurations and following structural optimizations without any constraint, we obtain a geometry similar to the washboard structure as shown in Fig. 1(a). However, different from the reported washboard structures of 2D group-VA systems, there is an additional atom at the center of the hexagon [which can be noticed from the top view in Fig. 1(a)] and, accordingly, we label this structure (belonging to the $\mathrm{Pbcm}$ space group) as a centered washboard (cw) to denote the difference. The unit cell of symmetric cw-borophene is rectangular with lattice constants of 1.61 and 5.11 A. The boron atoms form two different sublattices in this system at which atoms are puckered with a height $(\Delta h)$ of $1.48 \AA$. Also, boron atoms located at the same sublattice maintain a similar bond length pattern. Here, every $\mathrm{B}$ atom has six neighbors with three distinct bonds having lengths of $1.62,1.87$, and $1.90 \AA$. Next, we compute the cohesive energy (per B atom), which is defined as $E_{c}=\left(N * E[B]-E_{T}\right.$ [borophene] $) / N$, where $E[B], E_{T}$ [borophene], and $N$ represent the energy of a single $\mathrm{B}$ atom, the total energy of the optimized borophene structure, and the number of B atoms in the unit cell, respectively. $E_{c}$ is calculated as $5.72 \mathrm{eV} /$ atom, which is slightly less than what is reported for synthesized buckled borophene (5.74 eV/atom) [10]. Despite its relatively high $E_{c}, \mathrm{cw}-$ borophene is found to be unstable due to imaginary phonon modes that appear in the phonon dispersions. In the phonon band structure the soft longitudinal acoustic modes emerge near the $\Gamma$ point, as shown in Fig. 2(a). Eigenvectors, corresponding to these negative 


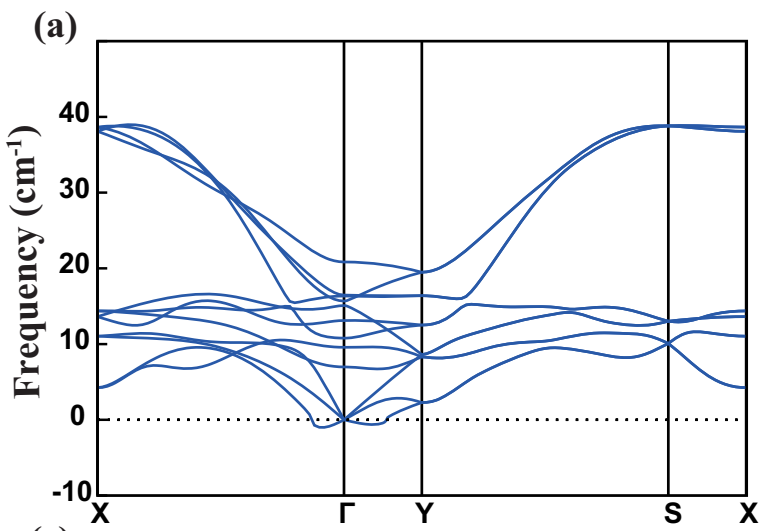

(c)
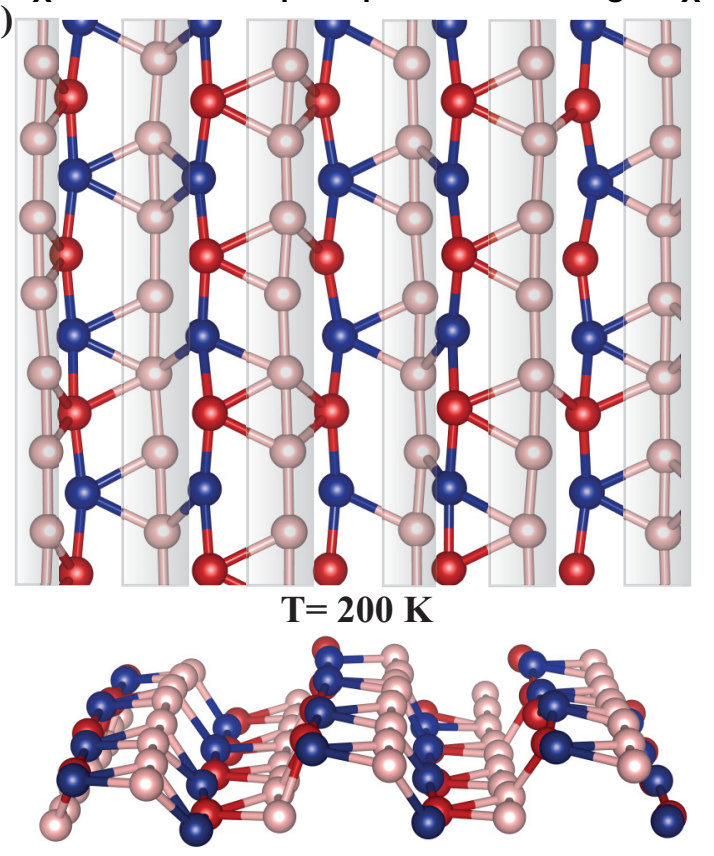

(b)

(d)
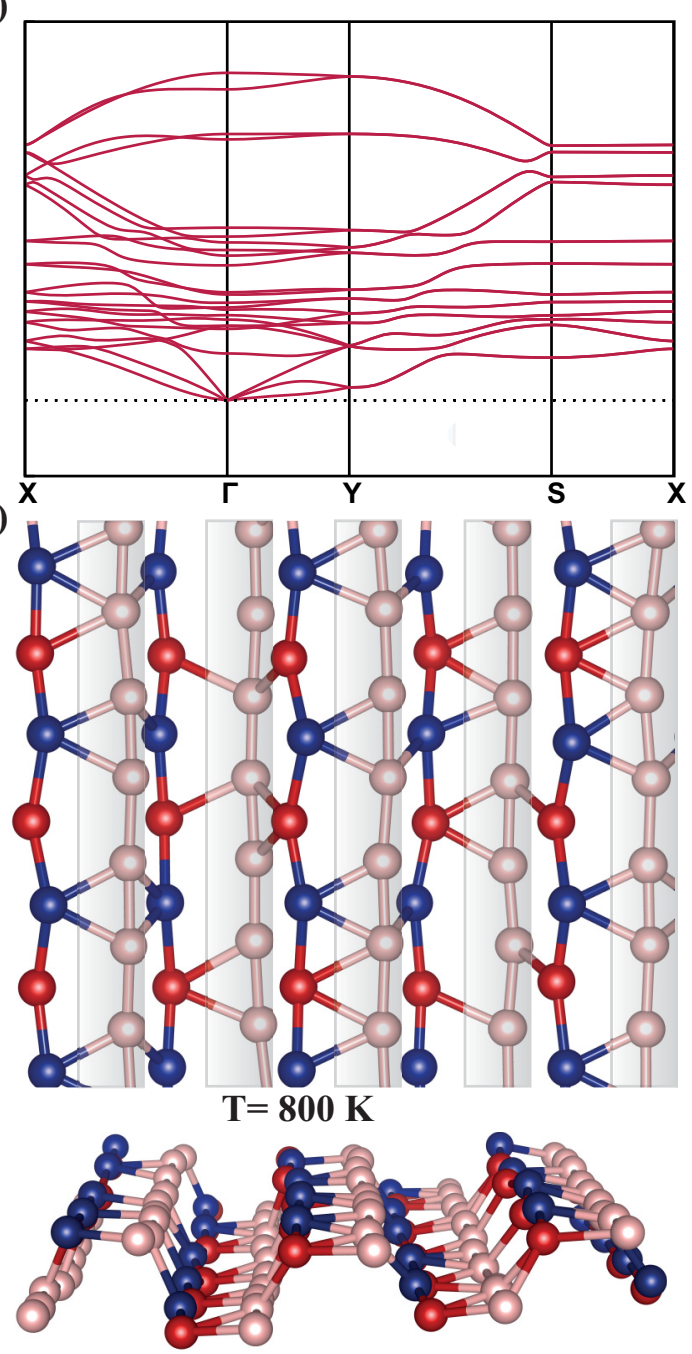

FIG. 2. The phonon spectrum of (a) cw- and (b) acw-borophene, and snapshots of molecular dynamics simulations of (c) cw-borophene at $200 \mathrm{~K}$ and (d) acw-borophene at $800 \mathrm{~K}$.

phonon modes, can be used to refine the ionic positions of B atoms by shifting their positions along the softest mode, which leads to a distortion in this case. Additionally, MD simulations (which are performed for a $12 \times 4$ supercell at 200, 400, 600, and $800 \mathrm{~K}$ for a total of $5 \mathrm{ps}$ simulation time) indicate that the structure of cw-borophene is maintained up to $800 \mathrm{~K}$ but with distortions along the vertical and horizontal directions starting from temperatures as low as $200 \mathrm{~K}$ [Fig. 2(c)].

The analysis of the phonon spectrum and MD simulations leads us to a different polymorph, namely, asymmetric centered-washboard (acw) borophene which is obtained with a $2 \times 1$ supercell allowing for the necessary reconstruction [Fig. 1(b)]. The unit cell of acw-borophene is also rectangular with lattice constants of 3.19 and $5.3 \AA$. B atoms in acwborophene have a significantly different bonding scheme than the symmetric geometry, where the bond lengths interchangeably differ even at the same sublattice (also quantified with bond order data) as shown in Fig. 1(b). Furthermore, there are two different $\Delta h$ values, 1.26 and $2.02 \AA$ depending on the position of the $\mathrm{B}$ atom, indicating a varying hybridization of B atoms. acw-borophene has orthorhombic symmetry and belongs to the $P m n 2_{1}$ space group, which is different than other recently proposed Pmmn and 8-Pmmn borophene systems [9,34]. $E_{c}$ is calculated as $5.73 \mathrm{eV} /$ atom, which is lower than the bulk boron but within the range of other reported 2D boron polymorphs $[9,33,35]$. When compared, $E_{c}(\mathrm{acw})$ is slightly higher than $(\sim 10 \mathrm{meV} /$ atom $) E_{c}(\mathrm{cw})-$ borophene. The increase in $E_{c}$ (or decrease in total energy) upon distortion signals the possibility of Peierls instability (see below). The optimized structure of acw-borophene has no negative frequencies in the phonon spectrum and the system is thermodynamically stable even at high temperatures up to $800 \mathrm{~K}$, as presented in Fig. 2(d). These results confirm that acw-borophene is not a metastable configuration but corresponds to a stable local minimum in the Born-Oppenheimer energy surface. The structural parameters and crystallographic positions of the cW- and acw-borophene are reported in the Supplemental Material [36]. 

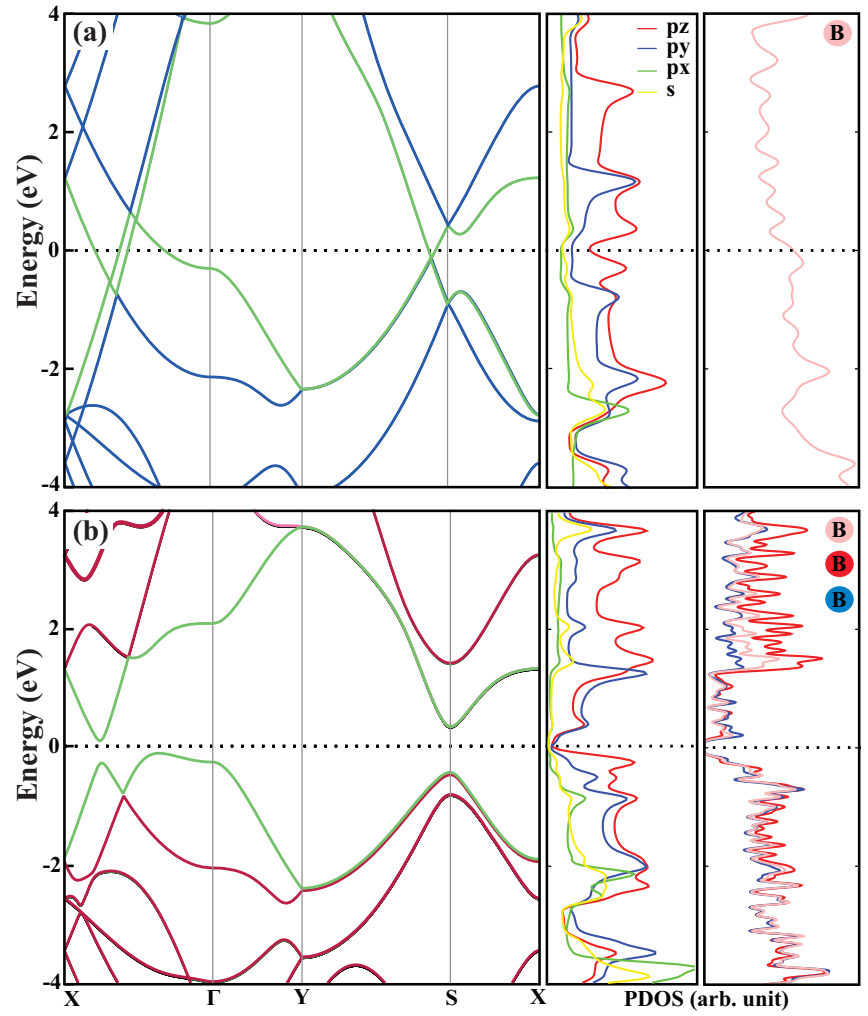

FIG. 3. Electronic structure of (a) cw- and (b) acw-borophene with orbital- and atomic-resolved partial density of states (PDOS). The bands which split upon distortion are shown with green solid lines. The Fermi level $\left(E_{F}\right)$ is set to zero and is shown by dotted lines.

Revealing the structural properties and stability, we examine the electronic structures of the considered systems. The analysis of the band structures of cw- and acw-borophene can elucidate the origin of the structural reconstruction. The band structure of cw-borophene is metallic with Dirac-like cones, mostly composed of $p_{z}$ orbitals and the contributions of each $\mathrm{B}$ atom are the same [Fig. 3(a)]. The cone along the $X-G$ and $Y-S$ lines is 0.42 and $0.09 \mathrm{eV}$ below $E_{F}$. The downward shift of the Dirac cones indicates an excess of electrons in cw-borophene, unlike the $\beta_{12}$ sheet with vacancies [15]. Similar to buckled borophene, the electronic properties of $\mathrm{cw}$-borophene are anisotropic and the system is metallic along the $X-G$ and $Y-S$ directions where the Dirac cones are located. Interestingly, acw-borophene is a semiconductor with an indirect band gap of $0.39 \mathrm{eV}$ along the $X-G$ direction, as shown in Fig. 3(b). Band crossings and splitting around the $E_{F}$ are shown with green solid lines. Additionally, as shown in the atomic-resolved partial density of states (PDOS), the attribution of distorted boron atoms to the band structure is not equivalent [Fig. 3(b)]. Subsequent to the DFT-PBE results, the electronic band structures are calculated with hybrid functionals (HSE06) [31] to further confirm the obtained results. The resulting band structures with HSE06 have the same character, only the band gap increases to $0.69 \mathrm{eV}$ [36]. This metal to semiconductor transition indicates that acwborophene is stabilized with a structural reconstruction due to a Peierls distortion and the band-gap opening reduces the total
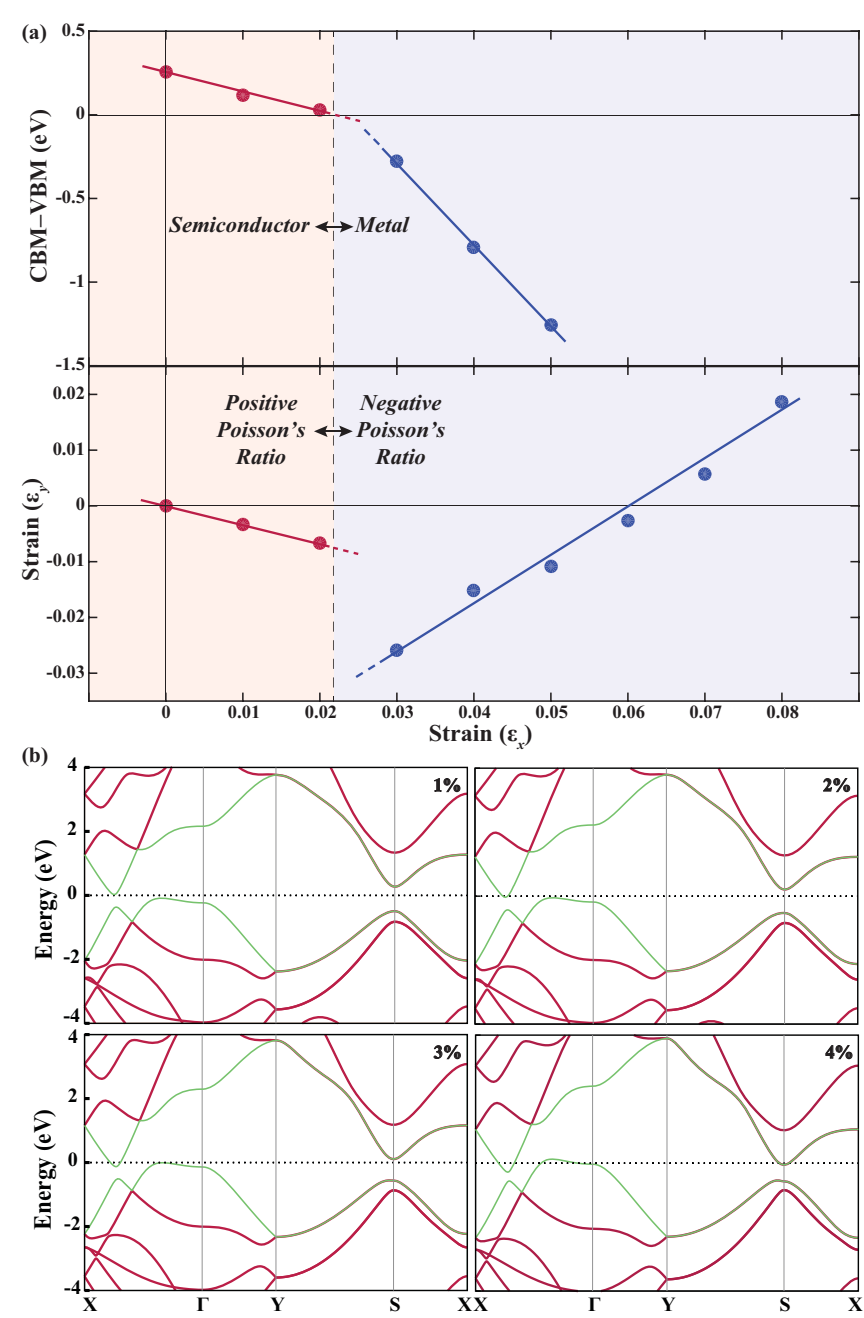

FIG. 4. (a) The variation of the electronic energy band gap and the equilibrium strain along the $y$ direction $\left(\epsilon_{y}\right)$ with respect to applied strain along the $x$ direction $\left(\epsilon_{x}\right)$. The band-gap values are extended to the metallic state by following the difference between the minimum of the conduction band (CBM) and the maximum of the valence band (VBM) after the transition. (b) The variations in the electronic band structure of acw-borophene upon applied uniaxial strain along $\epsilon_{x}$.

energy of the system. The Peierls instability for 1D metals has been recently extended for 2D systems with the concept of charge density waves induced by the nesting of Fermi surfaces $[12,37,38]$. It should be also noted that acw-borophene is the only semiconductor polymorph of borophene reported so far with no vacancies/defects.

Finally, we examine the effect of tensile strain on acwborophene to mimic lattice-mismatched substrates, and also it has been known that the properties of $2 \mathrm{D}$ materials can be tuned by strain engineering [39]. We investigate uniaxial strain up to $8 \%$, which covers the low and high tensile strain levels. As shown in Fig. 4(a), the gap between the conduction band minimum (CBM) and valence band maximum (VBM) gradually decreases with increasing strain and totally closes at $\sim 3 \%$. Interestingly, at this strain level distortion by virtue of the Peierls instability is removed and the system 
transforms back into symmetric $\mathrm{cw}$-borophene. This indicates that there is a trade-off between structural deformation and energy gained due to the band-gap opening, and once the gap closes, the structural phase transition simultaneously accompanies an electronic phase transition. Moreover, the Peierls instability not only affects the electronic properties of the system but also drastically changes the mechanical response. As shown in Fig. 4(b), for acw-borophene, while strain increases along the $x$ direction $\left(\epsilon_{x}\right)$, it tends to decrease along the transverse direction $\left(\epsilon_{y}\right)$, indicating a positive Poisson's ratio $\left(v=-\epsilon_{y} / \epsilon_{x} \approx 0.35\right.$ ). On the other hand, for $\epsilon_{x} \approx 0.03$ where the gap closes and distortion is altered, $\epsilon_{y}$ abruptly starts to increase with increasing $\epsilon_{x}$, making $v$ negative $(-0.25)$. This suggests that $\mathrm{cw}$-borophene, which can be stabilized with strain, has an intrinsic auxetic behavior similar to phosphorene [40] and graphene with vacancies [41]. Negative $v$ values have been also reported for other metallic phases of borophene [10], but the magnitudes are much smaller compared to the one that is calculated for cw-borophene. Similar results are obtained when calculations are repeated with biaxial strain instead of uniaxial strain [36].

In conclusion, we predict a semiconducting stable phase of borophene without defects, namely, an asymmetric centeredwashboard (acw) structure which is formed by structural reconstructions due to a Peierls instability in two dimensions. The stability of the aforementioned polymorph is provided in combination of the puckered geometry and Peierls distortion. The symmetric (cw) metallic system is sensitive to the Peierls instability and acw-borophene becomes a semiconductor following a structural transformation. Applying uniaxial or biaxial tensile strain to acw-borophene gradually lowers the band gap, and once it is fully closed, the system transforms back to the symmetric metallic state. Interestingly, the transformation not only affects the electronic structure but also drastically modifies the mechanical response in parallel. While the system exhibits a positive Poisson's ratio in the semiconducting regime, Poisson's ratio abruptly becomes negative once the system becomes metallic. The prediction of a stable semiconducting polymorph of borophene without defects and tunability of its electronic and mechanical response with strain can extend the usage of boron sheets in a variety of nanoelectronic applications.

This work was supported by the Scientific and Technological Research Council of Turkey (TUBITAK) under Project No. 115F088. The calculations were performed at TUBITAK ULAKBIM, High Performance and Grid Computing Center (TR-Grid e-Infrastructure) and the National Center for High Performance Computing of Turkey (UHeM) under Grant No. 5003622015. E.D. acknowledges support from The Turkish Academy of Sciences - Outstanding Young Scientists Award Program (TUBA-GEBIP).
[1] A. K. Singh, A. Sadrzadeh, and B. I. Yakobson, Nano Lett. 8, 1314 (2008).

[2] H.-J. Zhai, Y.-F. Zhao, W.-L. Li, Q. Chen, H. Bai, H.-S. Hu, Z. A. Piazza, W.-J. Tian, H.-G. Lu, Y.-B. Wu et al., Nat. Chem. 6, 727 (2014).

[3] Y. Yang, Z. Zhang, E. S. Penev, and B. I. Yakobson, Nanoscale 9, 1805 (2017).

[4] W.-L. Li, T. Jian, X. Chen, T.-T. Chen, G. V. Lopez, J. Li, and L.-S. Wang, Angew. Chem. 128, 7484 (2016).

[5] Z. A. Piazza, H.-S. Hu, W.-L. Li, Y.-F. Zhao, J. Li, and L.-S. Wang, Nat. Commun. 5, 3113 (2014).

[6] W. Huang, A. P. Sergeeva, H.-J. Zhai, B. B. Averkiev, L.-S. Wang, and A. I. Boldyrev, Nat. Chem. 2, 202 (2010).

[7] E. S. Penev, S. Bhowmick, A. Sadrzadeh, and B. I. Yakobson, Nano Lett. 12, 2441 (2012).

[8] H. Tang and S. Ismail-Beigi, Phys. Rev. Lett. 99, 115501 (2007).

[9] X.-F. Zhou, X. Dong, A. R. Oganov, Q. Zhu, Y. Tian, and H.-T. Wang, Phys. Rev. Lett. 112, 085502 (2014).

[10] A. J. Mannix, X.-F. Zhou, B. Kiraly, J. D. Wood, D. Alducin, B. D. Myers, X. Liu, B. L. Fisher, U. Santiago, J. R. Guest et al., Science 350, 1513 (2015).

[11] B. Feng, J. Zhang, Q. Zhong, W. Li, S. Li, H. Li, P. Cheng, S. Meng, L. Chen, and K. Wu, Nat. Chem. 8, 563 (2016).

[12] X. Liu, Z. Zhang, L. Wang, B. I. Yakobson, and M. C. Hersam, Nat. Mater. 17, 783 (2018).

[13] Z. Zhang, E. S. Penev, and B. I. Yakobson, Nat. Chem. 8, 525 (2016).

[14] M. Gao, Q.-Z. Li, X.-W. Yan, and J. Wang, Phys. Rev. B 95, 024505 (2017).
[15] B. Feng, O. Sugino, R.-Y. Liu, J. Zhang, R. Yukawa, M. Kawamura, T. Iimori, H. Kim, Y. Hasegawa, H. Li et al., Phys. Rev. Lett. 118, 096401 (2017).

[16] B. Peng, H. Zhang, H. Shao, Y. Xu, R. Zhang, and H. Zhu, J. Mater. Chem. C 4, 3592 (2016).

[17] B. Mortazavi, O. Rahaman, A. Dianat, and T. Rabczuk, Phys. Chem. Chem. Phys. 18, 27405 (2016).

[18] H. Sun, Q. Li, and X. Wan, Phys. Chem. Chem. Phys. 18, 14927 (2016).

[19] H. Xiao, W. Cao, T. Ouyang, S. Guo, C. He, and J. Zhong, Sci. Rep. 7, 45986 (2017).

[20] A. Lherbier, A. R. Botello-Méndez, and J.-C. Charlier, 2D Mater. 3, 045006 (2016).

[21] Z. Zhang, A. J. Mannix, Z. Hu, B. Kiraly, N. P. Guisinger, M. C. Hersam, and B. I. Yakobson, Nano Lett. 16, 6622 (2016).

[22] L. Adamska, S. Sadasivam, J. J. Foley, P. Darancet, and S. Sharifzadeh, J. Phys. Chem. C 122, 4037 (2018).

[23] Y. Chen, G. Yu, W. Chen, Y. Liu, G.-D. Li, P. Zhu, Q. Tao, Q. Li, J. Liu, X. Shen, H. Li, X. Huang, D. Wang, T. Asefa, and X. Zou, J. Am. Chem. Soc. 139, 12370 (2017).

[24] H. Zhou, Y. Cai, G. Zhang, and Y.-W. Zhang, npj 2D Mater. Appl. 1, 14 (2017).

[25] X. Liu, Z. Wei, I. Balla, A. J. Mannix, N. P. Guisinger, E. Luijten, and M. C. Hersam, Sci. Adv. 3, e1602356 (2017).

[26] W. Kohn and L. J. Sham, Phys. Rev. 140, A1133 (1965).

[27] P. Hohenberg and W. Kohn, Phys. Rev. 136, B864 (1964).

[28] G. Kresse and J. Furthmüller, Phys. Rev. B 54, 11169 (1996).

[29] P. E. Blöchl, Phys. Rev. B 50, 17953 (1994).

[30] J. P. Perdew, K. Burke, and M. Ernzerhof, Phys. Rev. Lett. 77, 3865 (1996). 
[31] J. Heyd, G. E. Scuseria, and M. Ernzerhof, J. Chem. Phys. 118, 8207 (2003).

[32] A. Togo and I. Tanaka, Scr. Mater. 108, 1 (2015).

[33] X. Wu, J. Dai, Y. Zhao, Z. Zhuo, J. Yang, and X. C. Zeng, ACS Nano 6, 7443 (2012).

[34] A. Lopez-Bezanilla and P. B. Littlewood, Phys. Rev. B 93, 241405(R) (2016).

[35] F. Ma, Y. Jiao, G. Gao, Y. Gu, A. Bilic, Z. Chen, and A. Du, Nano Lett. 16, 3022 (2016).

[36] See Supplemental Material at http://link.aps.org/supplemental/ 10.1103/PhysRevB.98.241408 for the cell parameters and atomic positions of $\mathrm{cw}$ - and acw-borophene; electronic band structures obtained with HSE06 under uniaxial strain; and electronic band structures under biaxial strain .
[37] S. Borisenko, A. Kordyuk, A. Yaresko, V. Zabolotnyy, D. Inosov, R. Schuster, B. Büchner, R. Weber, R. Follath, L. Patthey et al., Phys. Rev. Lett. 100, 196402 (2008).

[38] M. D. Johannes and I. I. Mazin, Phys. Rev. B 77, 165135 (2008).

[39] S. Cahangirov, H. Sahin, G. Le Lay, and A. Rubio, Strain engineering of 2D materials, in Introduction to the Physics of Silicene and other 2D Materials, Lecture Notes in Physics, Vol. 930 (Springer, Cham, 2017), pp. 87-96.

[40] H. Wang, X. Li, P. Li, and J. Yang, Nanoscale 9, 850 (2017).

[41] J. N. Grima, S. Winczewski, L. Mizzi, M. C. Grech, R. Cauchi, R. Gatt, D. Attard, K. W. Wojciechowski, and J. Rybicki, Adv. Mater. 27, 1455 (2015). 